\title{
MODELAGEM EMPIRICA PARA MAXIMIZAÇÃO DAVAZÃO DE ALIMENTAÇÃO DE UMA COLUNA FRACIONADORA DE PROPENO
}

\author{
M. Q. ARAUJO ${ }^{1}$, A. M. F. FILETI ${ }^{2}$, S. B. NEVES ${ }^{3}$ \\ ${ }^{1,2}$ Universidade Estadual de Campinas, Departamento de Engenharia de Sistemas Químicos. \\ ${ }^{3}$ Universidade Salvador, Departamento de Engenharia Quimica \\ E-mail para contato: marcia.queiroz.araujo@gmail.com
}

RESUMO - Este trabalho apresenta o desenvolvimento de uma metodologia para o cálculo direto da razão de refluxo e da vazão máxima de alimentação de uma Coluna Fracionadora de Propeno, através da regressão de modelos empíricos obtidos a partir de dos resultados rigorosos de programas comerciais de simulação estacionária e avaliação de equipamentos. Um cálculo rápido da vazão máxima de alimentação é uma ferramenta fundamental na implantação de sistemas de controle para otimização das condições operacionais da coluna. Logo, o interesse por estes resultados se deve à obtenção de uma operação mais rentável de uma coluna de alta pureza pelo alcance dos seus limites operacionais. Os resultados dos modelos propostos estão em plena concordância com os obtidos em programas comerciais de simulação de processos de destilação, de hidráulica de pratos e de troca térmica, com a vantagem de serem obtidos em menor tempo de computação e sem problemas de convergência numérica.

\section{I NTRODUÇÃO}

O presente estudo focaliza sua atenção na simulação das condições de operação de uma Coluna Fracionadora de Propeno de unidade de produção de olefinas. Em uma operação típica desta coluna, deseja-se estabelecer uma vazão de alimentação próxima à máxima permitida pelo sistema, a qual está condicionada a limitações de hidráulica da coluna e de troca térmica dos condensadores e refervedores. Um cálculo rigoroso destes limites para cada situação, usando programas de simulação comerciais, é trabalhoso e consome bastante tempo. Para contornar estas dificuldades, este trabalho propõe uma modelagem empírica semi-fenomenológica para o cálculo das máximas vazões de alimentação e de produtos possíveis para este equipamento e para cada caso específico formado por um conjunto de condições envolvendo variações na composição de propano na carga, composição de propeno no produto de fundo, pressão de operação, temperatura da água de resfriamento e coeficiente de incrustação do condensador.

\section{DESCRIÇÃO DO PROCESSO DA FRACIONADORA DE PROPENO}

A Coluna Fracionadora de Propeno tem como função a separação do propeno de outros hidrocarbonetos, onde o principal deles é o propano, tendo como vazão de alimentação uma 
corrente do corte $\mathrm{C}_{3}$ rica em propeno, a qual é proveniente do topo de uma coluna que separa a corrente $\mathrm{C}_{3}$ de oligômeros indesejáveis, conforme resultados de analises laboratoriais desta corrente. A Figura 1 representa esta coluna e seus principais equipamentos periféricos. A Tabela 1 apresenta as principais características operacionais e dimensionais deste equipamento.

Tabela 1 - Características operacionais e dimensionais da Coluna Fracionadora de Propeno

\begin{tabular}{|l|c|}
\hline Número de pratos & 159 \\
\hline Prato de alimentação & 80 \\
\hline Tipo de prato & Valvulado \\
\hline Número de passes dos pratos & 2 \\
\hline Diâmetro (m) & 4,8 \\
\hline Pressão de operação (kgf/cm ${ }^{2}$ man) & 14 a 19 \\
\hline $\begin{array}{l}\text { Especificação do propeno no topo } \\
\text { (\% massa) }\end{array}$ & 99,5 \\
\hline $\begin{array}{l}\text { Concentração de propeno no fundo } \\
(\% \text { massa) }\end{array}$ & 2 a 10 \\
\hline $\begin{array}{l}\text { Composição de propeno na carga } \\
(\% \text { massa) }\end{array}$ & 93 a 97 \\
\hline
\end{tabular}

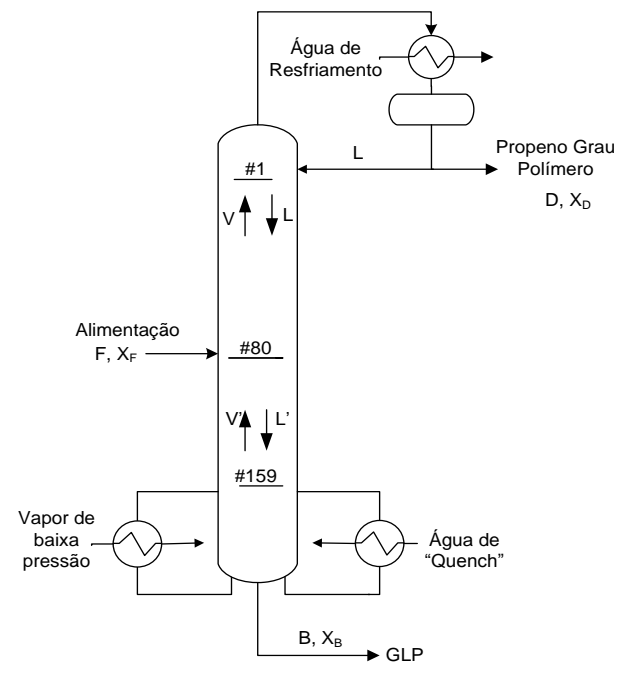

Figura 1. Representação da Coluna Fracionadora de Propeno

\section{METODOLOGIA PARA MODELAGEM DO PROCESSO}

A premissa adotada neste estudo da Coluna Fracionadora de Propeno corresponde a uma situação real industrial, na qual é disponível uma corrente de $\mathrm{C}_{3}$ rica em propeno com uma vazão maior do que a Coluna Fracionadora pode processar. Desta forma, considerando que o Propeno Grau Polímero(PP) produzido tem maior valor agregado que a corrente de $\mathrm{C}_{3}$ alimentada, é importante determinar a máxima vazão de alimentação possível para a coluna em cada situação específica. Um sistema de controle para otimização desta coluna determinando suas condições operacionais requer o desenvolvimento de modelos preditivos que represente cada cenário operacional, definindo valores de cada uma das variáveis anteriormente citadas.

O objetivo deste trabalho é o desenvolvimento destes modelos empíricos e, com estes modelos, determinar através de uma rotina matemática, os seguintes resultados para a coluna: Razão de refluxo (RR); Razões produtos/alimentação (B/F, L/F ou L'/F); Vazões máximas de alimentação na coluna $\left(\mathrm{F}_{\max }\right)$ para os seguintes limites ou restrições (carga térmica do condensador; inundação do prato por arraste (AA); inundação do prato por downcomer (DC). Estes resultados são definidos em função das seguintes variáveis: Composição (\% em massa) de propano da alimentação $\left(\mathrm{X}_{\mathrm{F}, 2}\right)$; Composição (\% em massa) de propeno no produto de fundo $\left(\mathrm{X}_{\mathrm{B}, 1}\right)$; Pressão de topo da coluna $(\mathrm{Pt})$; Temperatura entrada de água de resfriamento no condensador $\left(\mathrm{t}_{1}\right)$; Coeficiente de incrustação do condensador $\left(\mathrm{r}_{\mathrm{D}}\right)$. Para efeito de equacionamento 
e apresentação de resultados, foi adotado o índice 1 para o componente propeno, e o índice 2 para o componente propano.

\section{DESENVOLVIMENTO DOS MODELOS EMPÍRICOS}

O primeiro passo consiste na determinação das variáveis intensivas associadas à operação da coluna em cada condição, como a $R R$ e as razões B/F, L/F ou L'/F. Estas variáveis não dependem da vazão de alimentação, mas apenas dos seguintes parâmetros já mencionados: $\mathrm{X}_{\mathrm{F}, 2}$, $\mathrm{X}_{\mathrm{B}, 1}$ e Pt. Definida a RR, deve ser calculada a máxima vazão que pode alimentar a coluna, devido a cada um dos limites identificados: carga térmica do condensador, hidráulica dos pratos da coluna, inundação do DC e inundação da AA.

\subsection{Modelo Empírico Para Separação na Coluna Fracionadora de Propeno}

A separação na Coluna Fracionadora de Propeno é caracterizada por sua RR em cada caso estudado. Para que a função de RR empírica represente fielmente os resultados rigorosos do simulador comercial, foram efetuadas 150 simulações rigorosas levando em consideração os parâmetros que influenciam na RR. Desta forma, foram realizadas simulações das combinações de cinco valores de $X_{\mathrm{F}, 2}(3 \%, 4 \%, 5 \%, 6 \%$ e $7 \%)$, cinco valores de $X_{\mathrm{B}, 1}(2 \%, 4 \%, 6 \%, 8 \%$ e $10 \%)$ e seis valores $\mathrm{Pt}\left(14,15,16,17,18\right.$ e $19 \mathrm{kgf} / \mathrm{cm}^{2}$ man). Uma composição típica da alimentação da coluna é apresentada na Tabela 2. Operacionalmente, a variação da composição de alimentação é caracterizada pela variação da $X_{F, 2}$. A composição de propeno na alimentação $\left(X_{F, 1}\right)$, por exemplo, é calculada através da Equação 1.

$$
X_{F, 1}=\frac{0,9367579}{0,9396718} \cdot\left(1-X_{F, 2}\right) \quad(1)
$$

Tabela 2. Composição típica da alimentação da Coluna Fracionadora de Propeno

\begin{tabular}{|c|c|c|c|c|c|}
\hline $\begin{array}{l}\text { Componen- } \\
\text { tes }\end{array}$ & $\begin{array}{c}\% \\
\text { mássic } \\
\text { a }\end{array}$ & $\begin{array}{c}\text { Componen- } \\
\text { tes }\end{array}$ & $\begin{array}{c}\% \\
\text { mássica }\end{array}$ & $\begin{array}{c}\text { Componen- } \\
\text { tes }\end{array}$ & $\begin{array}{c}\% \\
\text { mássica }\end{array}$ \\
\hline Hidrogênio & 0,0012 & Propeno & 93,6758 & Buteno 1 & 0,0414 \\
\hline Vinil Acetileno & 0,0001 & Propano & 6,0328 & Butadieno & 0,1181 \\
\hline Eteno & 0,0003 & i-Butano & 0,0532 & n-Butano & 0,0020 \\
\hline Etano & 0,0092 & i-Buteno & 0,0632 & Buteno 2 & 0,0021 \\
\hline \multicolumn{5}{|l|}{ Monóxido de carbono } & 0,0008 \\
\hline
\end{tabular}


As simulações foram efetuadas considerando que a Pt é a pressão no vaso de refluxo, e uma perda de carga de $0,2 \mathrm{kgf} / \mathrm{cm}^{2}$ para o condensador, assim como $0,6 \mathrm{kgf} / \mathrm{cm}^{2}$ é a perda de carga da coluna, segundo dados operacionais.
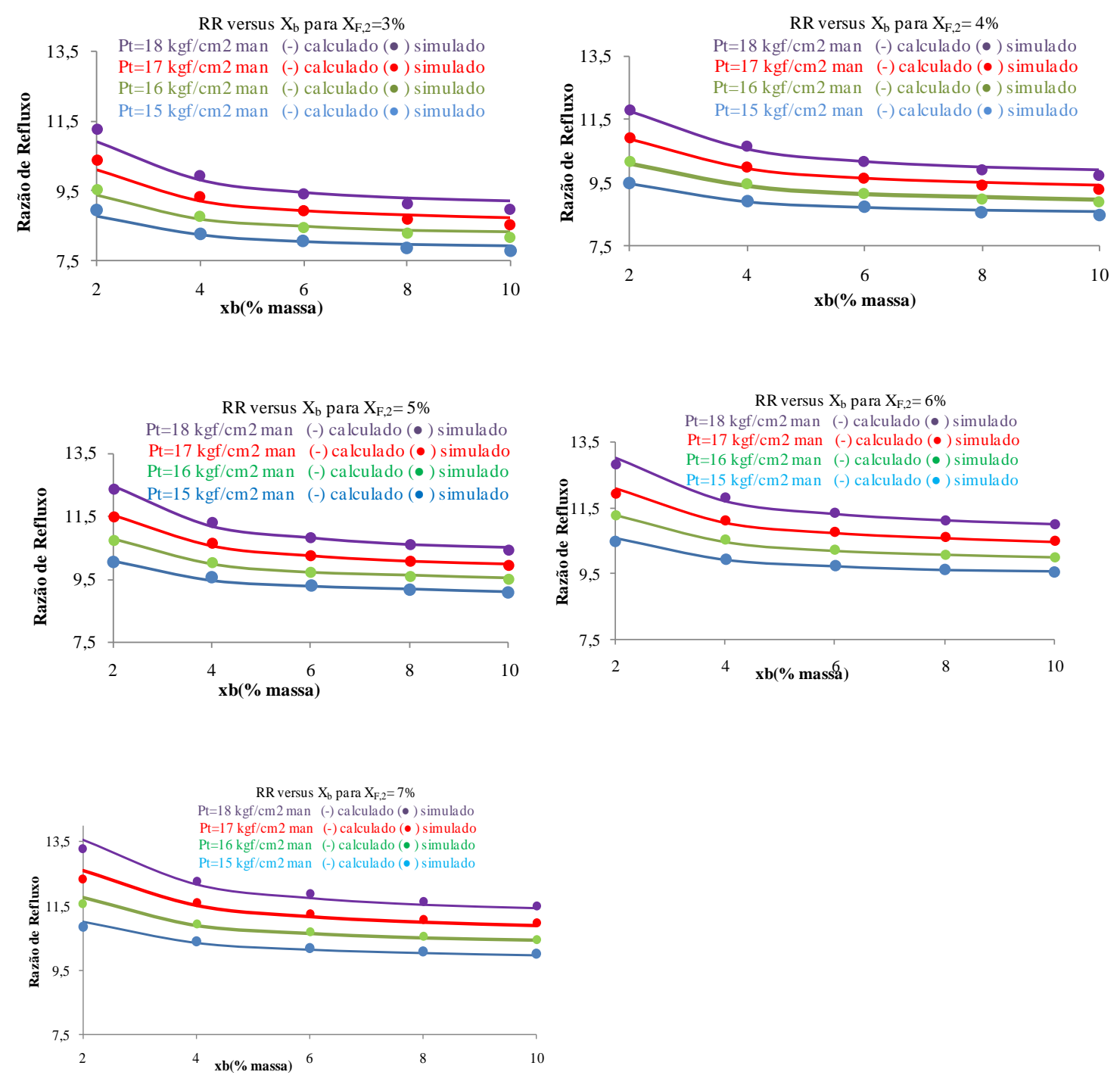

Figura 2. Razão de refluxo calculada e simulada em função da composição de propeno no fundo $\left(\mathrm{X}_{\mathrm{B}, 1}\right)$, para diversas composições de propano na carga $\left(\mathrm{X}_{\mathrm{F}, 2}\right)$ em \% massa, e pressões $(\mathrm{Pt})$ selecionadas no topo da coluna

Desta forma, para cada valor de pressão de topo foi obtido um conjunto de 25 dados de RR em função de $X_{F, 2}$ e $X_{B, 1}$. Este conjunto de dados é uma função de duas variáveis para a RR, que foi ajustada usando o programa LabFit, conforme SILVA e SILVA (2012) para uma equação do tipo mostrado na Equação 2. 
$R R=e^{\left(A+\frac{B}{X_{B, 1}}+C \cdot \ln X_{F, 2}\right)}$

$B=0,00328 \cdot P_{t}^{2}-0,050094 \cdot P_{t}+0,26260$ onde: $A=0,001775 \cdot P_{t}^{2}-0,008265 \cdot P_{t}+1,47079$

$$
C=-0,0007475 \cdot P_{t}^{2}+0,0191545 \cdot P_{t}+0,152889
$$

Comparando-se os resultados desta função com os da simulação rigorosa observa-se um erro relativo médio de $0,76 \%$, variando na faixa de $-3,4 \%$ a $2,8 \%$. Estes resultados são aceitáveis para uso em controle, onde os erros de medição de vazões são da ordem de $2 \%$. A Figura 2 apresenta comparativamente as RR calculadas com o modelo proposto e simuladas de forma rigorosa, para pressões na faixa de 15 a $18 \mathrm{kgf} / \mathrm{cm}^{2}$ man.

\subsection{Modelo Empírico para Vazão Máxima Limitada por Condensador}

Um balanço material para o propeno é feito no topo da coluna, conforme Equação 6, permitindo calcular a vazão de destilado máxima $\left(\mathrm{D}_{\max }\right)$. E, o cálculo da Fmax pode ser feito pela Equação 7.

$$
D_{\max }=\frac{V_{\max }}{(R R+1)} \quad(6) \quad F_{\max }=\frac{D_{\max } \cdot\left(0,995-X_{B, 1}\right)}{X_{F, 1}-X_{B, 1}} \quad(7) \quad V \max =V \max _{a p r} \cdot F c
$$

Para o cálculo da vazão máxima de condensação $\left(\mathrm{V}_{\max }\right)$, equação 8 , deve ser utilizado o procedimento iterativo a seguir, utilizando um fator de aproximação, Fc, determinado por regressão de dados simulados, consolidados pela equação 8.2:

1. Estima-se um valor para a Vmax, como primeira aproximação;

2. Calcula-se as temperaturas de saturação do produto de topo, na entrada e na saída do condensador $\left(\mathrm{T}_{\mathrm{C} 1}\right.$ e $\left.\mathrm{T}_{\mathrm{C} 2}\right)$ e também as diferenças de temperaturas no condensador. Sendo $\mathrm{t}_{1}$ e $\mathrm{t}_{2}$ as temperaturas de entrada e saída da AGR no condensador, respectivamente. Na primeira iteração $t_{2}$ pode ser estimado como o valor médio entre $T_{C 1}$ e $t_{1}$

3. Calcula-se os coeficientes de película médios no lado externo (PP condensando) e interno (AGR), desenvolvidos por regressão a partir de dados obtidos em simulador comercial rigoroso de trocadores de calor.

4. Calcular o valor do coeficiente global de troca térmica (U).

5. Calcular o valor da carga térmica (Q), a partir de dados resultantes de um simulador rigoroso.

6. Calcula-se o calor de condensação do propeno $(\triangle H)$ e o calor específico médio da água de resfriamento ( $\mathrm{Cp}_{\text {médio }}$ ) utilizando as equações de Daubert e Danner (1984).

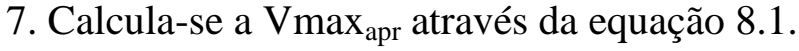

$$
V \text { max }_{a p r}=\frac{Q}{\Delta H} \quad(8.1)
$$

8. Calcula-se $t_{2}$ a partir de um balanço de energia traduzido pela Equação 9 e compara com $t_{2}$ da estimativa anterior. Proceder à convergência de $t_{2}$ retornando para a etapa 2 , se necessário.

$$
F c=1 /(1.6008+23.134 \cdot(\text { risuj }+ \text { rosuj })-9.411 /(P)) \quad(8.2) \quad t_{2}=t_{1}+\frac{Q}{\left(\text { Vazão }_{A G R} \cdot C p_{\text {medio }}\right)}
$$


Desta forma, os resultados de $\mathrm{F}_{\max }$ do modelo empírico concordam com os valores do simulador de trocador de calor dentro de um erro médio de 1,06\%, com erros máximos de 2,2\%.

\subsection{Modelo Empírico para Vazão Máxima Limitada por Hidráulica dos Pratos}

Analisando o escoamento de líquido e vapor nos pratos da coluna, verifica-se a existência de dois limites de capacidade: inundação da AA do prato por arraste e inundação do DC.

O cálculo da $\mathrm{F}_{\max }$ limitada pela inundação da $\mathrm{AA}\left(\mathrm{F}_{\max \_A A}\right)$ pode ser calculada na Equação 10. Para que seja resolvida a Equação 10, os seus termos devem ser determinados. Com esta finalidade foi estabelecido que a inundação máxima permitida nas operações, sem risco da perda de especificação do produto de topo da coluna, é de $95 \%$.

As equações do balanço material global da coluna e do balanço para propeno permitem calcular o termo B/F de acordo com a equação 11 .

$$
F_{\text {max } \_A A}=\frac{V_{A A}^{\prime}}{\left(L^{\prime} / F\right)-(B / F)}(10) \quad \frac{B}{F}=1-\left(\frac{\left(X_{F, 1}-X_{B, 1}\right)}{\left(0,995-X_{B, 1}\right)}\right)(11) \quad\left(\frac{L}{F}\right) \text { massico }=\left(\frac{\left(X_{F, 1}-X_{B, 1}\right)}{\left(0,995-X_{B, 1}\right)}\right) \cdot R R
$$

O termo L'/F é calculado, utilizando um modelo ideal, através das Equações 12 a 16. A premissa do modelo ideal adotado foi de vazões molares de líquido e vapor constantes em cada seção da coluna. Este valor de L'/F foi adicionalmente corrigido para refletir os resultados da simulação rigorosa. Esta correção deu-se através de uma função de $\mathrm{X}_{\mathrm{F}, 2}$ e $\mathrm{X}_{\mathrm{B}, 1}$ e, após correção, os erros médio e máximo foram de $0,81 \%$ e $2,55 \%$, respectivamente.

$$
\begin{aligned}
& \left(\frac{L}{F}\right)_{\text {molar }}=\left(\frac{L}{F}\right)_{\text {massico }} \cdot \frac{P M_{\text {topo }}}{P M_{\text {carga }}}(13) \quad\left(\frac{L^{\prime}}{F}\right)_{\text {molar }}=\left(\frac{L}{F}\right)_{\text {molar }}+1+\frac{C p_{\text {liqdealim }} \cdot\left(T_{\text {pratodealim }}-T_{\text {alim }}\right)}{\Delta H_{\text {vaporpratodealim }}} \\
& \left(\frac{L^{\prime}}{F}\right)_{\text {massico_ideal }}=\left(\frac{L^{\prime}}{F}\right)_{\text {molar }} \cdot \frac{P M_{\text {fundo }}}{P M_{\text {carga }}}
\end{aligned}
$$

A representação da Equação 16 considera uma alimentação de carga na coluna na condição de líquido subresfriado. Os valores de calor específico do líquido de alimentação (Cp $\mathrm{p}_{\text {liq }}$ de alim) e calor de condensação do vapor que chega ao prato de alimentação $\left(\triangle \mathrm{H}_{\text {vapor prato de alim) }}\right.$ foram calculados por equações segundo Daubert e Danner (1984).

A expressão da vazão mássica interna máxima ( $\left.\mathrm{V}^{\prime}{ }_{\mathrm{AA}}\right)$ que limita a operação da coluna, especificando o produto de topo sem que ocorra a inundação da AA, pode ser determinada através da equação da vazão volumétrica de vapor interna da coluna (V', volumetrico_AA ) e da massa específica da mistura gasosa, pela Equação 17. O cálculo da massa específica da mistura da fase vapor $\left(\rho_{V}\right)$ foi obtido pela utilização da equação de Clayperon para mistura de gás ideal, em função da pressão, temperatura e peso molecular. O ajuste da não idealidade foi efetuado com o 
cálculo do fator de compressibilidade (Z) e ajustado com os resultados do simulador por regressão de dados. Desta forma, foi obtida a expressão para a massa específica para a mistura gasosa real. A expressão para a vazão volumétrica (V'volumetrico_AA) é obtida através da correlação dos valores encontrados para V'volumétrico obtido por regressão dos dados simulados considerando $95 \%$ de inundação da área ativa e representada pela Equação 18. O membro da esquerda desta equação corresponde à constante de Souders \& Brown (CSB) descrita por Kister (1992).

$$
\begin{aligned}
& V_{A A}^{\prime}=V_{\text {VolumetricAA }}^{\prime} \rho_{V}(17) \\
& V_{\text {volumétrioAA }}^{\prime} \sqrt{\frac{\rho V}{(\rho L-\rho V)}}=-65,7799 \cdot\left(\frac{V^{\prime}}{L^{\prime}}\right)^{3}+2077,8851 \cdot\left(\frac{V^{\prime}}{L^{\prime}}\right)^{2}-21666,6396 \cdot\left(\frac{V^{\prime}}{L^{\prime}}\right)+77731,8323
\end{aligned}
$$

O cálculo da vazão máxima de alimentação da coluna limitada pela inundação por DC (Fmax_DC) pode ser feito através da Equação 19. O termo V'/F pode ser calculado pelo balanço material no fundo da coluna expresso pela Equação 20 e o valor de L'/F foi obtido da Equação 16.

$$
\operatorname{Fmax}_{D C}=\frac{L_{D C}^{\prime}}{\left(\frac{V^{\prime}}{F}\right)+\left(\frac{B}{F}\right)} \quad(19) \quad \frac{V^{\prime}}{F}=\frac{L^{\prime}}{F}-\frac{B}{F} \quad(20) \quad L_{D C}^{\prime}=L_{\text {volumetrio } D C}^{\prime} . \rho_{L}
$$

As vazões volumétrica e mássica de líquido (L') são obtidas através dos resultados do simulador por regressão de dados de L' da simulação rigorosa para a condição de $95 \%$ de inundação do downcomer em função de V'/L', vistos nas Equações 21 e 22.

$$
L_{\text {volumétrio } D C}^{\prime}=-0,95477 \cdot\left(\frac{V^{\prime}}{L^{\prime}}\right)^{3}+27,04594 \cdot\left(\frac{V^{\prime}}{L^{\prime}}\right)^{2}-256,42221 .\left(\frac{V^{\prime}}{L^{\prime}}\right)+1804,37387(22)
$$

\section{RESULTADOS DAS VAZÕES MÁXIMAS DE ALIMENTAÇÃO DA COLUNA FRACIONADORA}

Este trabalho tem como objetivo a identificação da $F_{\max }$ supondo existir excedente da corrente $\mathrm{C}_{3}{ }^{+}$, devido à redução do consumo de propeno grau químico. Com a determinação da função representativa da RR, as vazões internas V, V' e L e L' foram determinadas e comparadas com aquelas obtidas pelos simuladores comerciais e tratadas para garantir a consistência dos resultados, considerando a máxima inundação de 95\%. O cálculo da Fmax considera as restrições operacionais como inundação da coluna por AA ou do DC e a carga térmica admissível no condensador. restrição que produz o menor valor da $\mathrm{F}_{\max }$ em cada caso será a máxima $\mathrm{F}_{\max }$. Alguns dos resultados obtidos por esta ferramenta estão apresentados nas Figuras 3 e 4 . Para o caso em que $X_{F, 2}=3 \%$, com condensador no inicio de campanha, a Fmax é de 44,1 ton/h, o que pode ser obtido com $\mathrm{Pt}=14,8 \mathrm{kgf} / \mathrm{cm}^{2}$ e $\mathrm{X}_{\mathrm{B}, 1}=10 \%$. Estes resultados são apresentados na Figura 3. E para o caso em que o $\mathrm{X}_{\mathrm{F}, 2}=7 \%$, com condensador no final de campanha, a Fmax é de 30,1 
ton $/ \mathrm{h}$, o que pode ser obtido com $\mathrm{Pt}=16,1 \mathrm{kgf} / \mathrm{cm}^{2}$ e $\mathrm{X}_{\mathrm{B}, 1}=2 \%$. Estes resultados são apresentados na Figura 4.

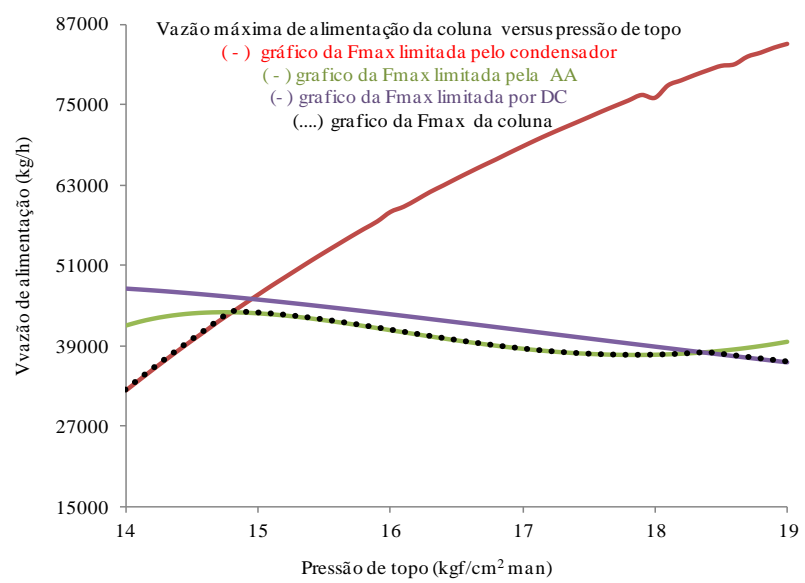

Figura 3. Vazão máxima da carga em função da Pt para $\mathrm{X}_{\mathrm{F}, 2}=3 \%, \mathrm{X}_{\mathrm{B}, 1}=10 \%, \mathrm{t}_{1}=28^{\circ} \mathrm{C}$ e condensador em inicio de campanha.

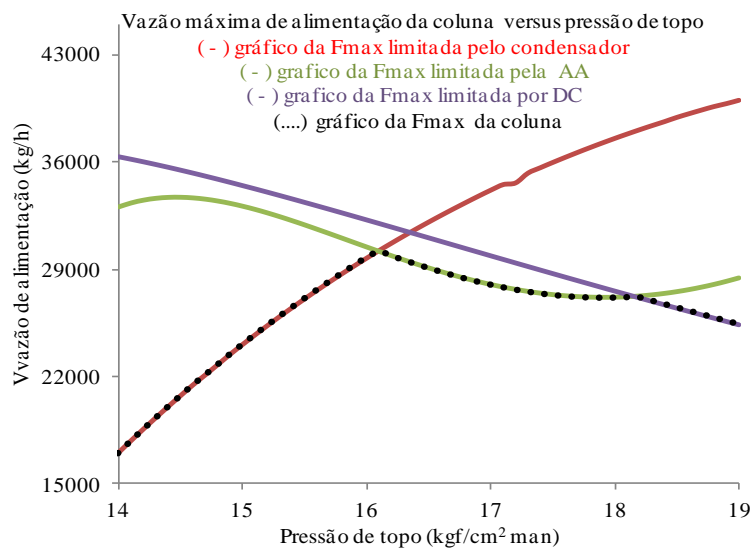

Figura 4. Vazão máxima da carga em da $\mathrm{Pt}$ para $\mathrm{X}_{\mathrm{F}, 2}=7 \%, \quad \mathrm{X}_{\mathrm{B}, 1}=2 \%, \quad \mathrm{t}_{1}=28^{\circ} \mathrm{C} \quad \mathrm{e}$ condensador no final de campanha.

\section{CONCLUSÃO}

Neste trabalho foi apresentada uma forma para obtenção da $\mathrm{F}_{\max }$ de uma fracionadora de propeno de alta pureza, considerando seus limites operacionais, balanços materiais e de energia. Os resultados obtidos pelo uso da ferramenta computacional desenvolvida são as $\mathrm{F}_{\max }$ em cada caso específico, com a vantagem de evitar cálculos que possam gerar problemas de convergência.

\section{REFERÊNCIAS}

DAUBERT, T. E.; DANNER, R. P. Data Compilation Tables of Properties of Pure Compounds. Design Institute for Physical Property Data. American Institute of Chemical Engineers, 1984.

KISTER, H. Z. Distillation Operation. Nova York: McGraw-Hill, 1990.

KISTER, H. Z., Distillation Design. Nova York: McGraw-Hill, 1992.

SILVA, W.; SILVA, C. P., Lab Fit - Software de Ajustes de Curvas. Disponível em:< http://zeus.df.ufcg.edu.br/labfit/index_p.htm . Acesso em: 11 jul.2012. 